\title{
Trossero tango therapy and psychological distress reduction in female cancer patients: An Italian pilot study
}

\author{
Costantini $\mathrm{A}^{1}$, Mazzotti $\mathrm{E}^{2 *}$, Brunetti $\mathrm{S}^{1}$, Navarra $\mathrm{C}^{1}$, Marchetti $\mathrm{P}^{3}$ and Trossero $\mathrm{F}^{4}$ \\ ${ }^{1}$ Psycho-Oncology Departmental Unit, Sant' Andrea Hospital, Sapienza University of Rome, Italy \\ ${ }^{2}$ Department of Clinical and Molecular Medicine, Sant' Andrea Hospital, Sapienza University of Rome, Italy \\ ${ }^{3}$ Medical Oncology, Sapienza University of Rome, Italy \\ ${ }^{4}$ Centro de Tango terapia, Rosario, Argentina
}

\begin{abstract}
Objectives: To estimate the effect of an 8-week Tango-based Therapy (TT) in reducing psychological distress among cancer patients

Design: To assess the change at different point of the study an estimate of the effect-size according to Cohen's coefficient $d$ was adopted.

Participants: Sixty-four female cancer patients were assigned to a TT group or to a waiting list (WL).

Setting: Classes of TT were held in a spacious room at a large hospital in Rome, Italy. Intervention. Participants completed a time-limited format of eight-weekly 90-minute sessions, in a small group setting (9-15 participants) conducted by two female psychotherapists.

Main outcome measures: Psychological distress, anxiety and depression, quality of life, and feelings about one's body were assessed at baseline and 8 weeks later.

Results: Forty-seven patients completed both assessments. Changes, measured as effect sizes, were reported by TT group patients in Distress (d=0.53), Anxiety $(\mathrm{d}=0.34)$, QOL-social ( $\mathrm{d}=0.37)$, QOL-emotional $(\mathrm{d}=0.43)$, QOL-role $(\mathrm{d}=0.33)$, and in feeling one's body as source of pleasure ( $\mathrm{d}=0.14)$ and in body's regained sensuality $(\mathrm{d}=0.18)$ has been reported.

Conclusions: The Tango-based Therapy has showed an impact on patients' wellbeing, has promoted self-acceptance, has enabled contact with one's body, and has allowed to be touched by the other. As it is important to offer psychological support to cancer patients, TT is characterized as an intervention accessible to many, and may be more acceptable even to people who are less receptive to psychological interventions.
\end{abstract}

\section{Introduction}

Neuroscience studies have confirmed that physical exercise increases the levels of BDNF (Brain-Derived Neurotrophic Factor) entailed in the mechanisms of synaptic plasticity, survival and differentiation of neurons, while substances such as serotonin and dopamine are released which influence mood, sense of vitality, activation and feelings of well-being [1]. Results of clinical research support the effectiveness of the physical movement to relieve fatigue, in patients with solid tumours $[2,3]$, and the dance and music effects on emotional, physical and mental state $[4,5]$.

Dance is a universal human behavior, often associated with group rituals, thousands of years old, so much so that it appears in the cave paintings of about 20,000 years ago. But it is also a complex sensorimotor action that metabolically activates the brain [6]. Ballroom dancing and belly dance adapted for cancer patients can be a viable form of physical activity with benefits for quality of life [5,7-10]. Music therapy is associated with an improvement in the mood of depressed patients and decreased pain intensity in cancer patients [11,12]. Emotions evoked by music can modulate the activity in the limbic and paralimbic brain structures with interesting implications for therapeutic approaches in the treatment of affective disorders [4,13]. Listening to a piece of modern Argentine tango affects the large-scale neural networks and activates the motor, emotional and creative areas of the brain [6,14]. Dancing Argentine tango has positive emotional effects: it reduces cortisol, depression and insomnia and could be included in stress management programmes $[15,16]$. Argentine tango has been applied to treat depression and stress; its effects have been compared to mindfulness and waiting list. Patients in Argentine tango and mindfulness groups reported reduced levels of depression, as compared to waiting list patients; stress levels were significantly reduced only in the Argentine tango group [15].

Although physical movement, music and dance have proven effective in alleviating some treatment-related symptoms in cancer patients, the multidimensionality of cancer distress has required clinicians and researchers to integrate specific psychotherapeutic approaches to the therapeutic components of dance and movement. Dance Movement Therapy (DMT) emphasizes the interconnection between mind and body, and through the psychotherapeutic use

${ }^{\star}$ Correspondence to: Eva Mazzotti, Department of Clinical and Molecular Medicine, Sant'Andrea Hospital, Faculty of Medicine and Psychology, "La Sapienza” University of Rome. Via di Grottarossa, Rome, Italy, E-mail: eva.mazzotti@gmail.com

Key words: cancer, tango therapy, distress thermometer, group therapy, psychological distress

Received: May 07, 2020; Accepted: May 20, 2020; Published: May 25, 2020 
of movement promotes physical, emotional, cognitive and social integration of the individual with the objective of improving health and well-being [16-18]. Positive results on perceived distress and pain severity, after DMT, have been reported among cancer patients undergoing radiotherapy [18]. A recent Cochrane review on DMT provided by formally trained dance-movement therapist for cancer patients did not find evidence for effects on depression, anxiety, stress, fatigue and body image. The review suggests that DMT may have beneficial effects on quality of life, somatization and vigor among women with breast cancer, and the low drop-out rate indicates that DMT is well tolerated and accepted [8].

This body of scientific evidence together with the awareness that cancer patients go through an experience of illness that mortifies their vital emotions and their ability to still consider their body as a source of pleasure, hence undermining their capacity to express themselves and feel through their body, has motivated our hypothesis that a tango therapy, as a variant of DMT, could address psychological needs of cancer patients (i.e.: not being regarded as a person, loss of sexual function, fatigue, anger, anxiety) [19,20]. Argentine tango is particularly accepted and loved in Italian culture and was also included in the Italian guidelines on the rehabilitation of Parkinson's disease for its effectiveness on walking and mood $[21,22]$. According to simultaneous care programs, include mind-body group psychotherapy such as tango therapy $[23,24]$ is possible improve psycho-physical patients' wellbeing [8,25-27].

In this study we chose to test the effectiveness of a standardized time-limited clinical protocol for Tango-based therapy [23,24] by measuring its impact on patients' distress, quality of life and pleasure found in one's own body.

\section{Objectives}

Using a randomized wait list-control design, we aimed to measure effect of Tango-based therapy (TT) on psychological distress (primary) as well as anxiety, depression, quality of life, and feelings about one's body (secondary outcomes) among cancer survivors.

\section{Materials and methods}

This experimental study has been conducted in compliance with the Declaration of Helsinki (1964, revised in 2004). Study approval was granted by the independent ethics committee of the "la Sapienza" University of Rome (Prot. N.186 SA-2016 del 27.10.2016). Patients provided written informed consent to the collection and release of anonymized data.

The patients were recruited at the Division of Oncology of the hospital and approached during their session of chemotherapy. Patients with a life expectancy of less than 6 months were excluded.

A total of 72 breast cancer patients were randomly assigned to 8-weekly classes of TT $(\mathrm{N}=36)$ or to 8-week of waiting list (WL, $\mathrm{N}=36$ ). Classes of TT were held in a spacious room at a large hospital in Rome, Italy, in 3 successive groups $(n=15, n=12$ and $n=9$, respectively), over 24 weeks.

\section{Treatment}

Trossero tango therapy consists of an integrated mind-body group psychotherapy $[23,24]$. The elements the characterize that Argentine tango (dance, music, codes, and symbolism) act as intermediaries and facilitators of symbolic expression of emotions and experiences inherent in the relationship with one's body and the representation of oneself particularly mortified after oncological therapies.

Since 2006, Trossero has trained a large number of psychotherapists in Europe and Latin America, providing them with guidelines for method replication (standardized operational criteria).

Each 90-minute session is divided into standardized modules according to the following scheme:

welcome, and relaxation exercises (5'); three repetitions of Argentine tango exercises (10' each), intercalated by group debriefing (10' each). Each session end with a group elaboration of the entire experience (30'), a wrap-up (5') e an applause.

The Argentine tango exercises may concern learn and practice the basic steps of Argentine tango, the practice of the tango codes as "mirada y cabeceo", the listen the tango music and its poetry; interpret music freely with improvised tango movements, exercises of connection, communication and coordination, different experiences of the embrace, in a progression of physical contact up to the closed embrace, simple dance sequences with basic steps, in open or closed embrace and the turn and change of direction.

In this study a short and time-limited format of eight weekly 90 -minute sessions was used, in small groups ( $\mathrm{n}=9-15$ participants). To participate no dance skills required. Each group has been conducted by two female psychotherapists with a thirty-year experience in mental and oncological health. Both formed by Trossero according to the standardized model $[23,24]$, one of them with 10 years of Argentine tango experience.

Waiting list patients underwent visits according to clinical practice. They did not benefit from psychological support during the 8 weeks of the study. None of them practiced dance.

\section{Measures}

The Distress Thermometer [19] is a 1-item self-report screening tool for evaluating psychological distress in cancer patients, in the week before. Patient grades distress level on a vertical visual analogue scale (VAS) with scores from 0 (no distress) to 10 (extreme distress). A cut-off score $>5$ has been chosen as index of significant distress (sensitivity $=0.70$, specificity $=0.77$ ).

The EORTC QLQ-C30 [28] is a questionnaire developed to assess the quality of life (qol) in cancer patients. It comprises five functional scales: Physical ( 5 items), Role (2 items), Emotional ( 2 items), Cognitive (4 items) and Social (2 items). Nine cancer relevant symptoms (13 items), and one global qol score ( 2 items). The answers are coded on a scale with 1-4 scores.

The Hospital Anxiety and Depression Scale [29] is a questionnaire consisting of 14 items, seven for anxiety (Anxiety subscale; HADS-A) and seven for depression (Depression subscale; HADS-D). The answers are coded on a scale with $0-3$ scores (range $0-21$ ). Scores $>=11$ were chosen for caseness, for both HADS subscales (T score $>52.38$ ) [30,31].

Comfort, Pleasure, Sensuality, and Passion about one's body, 4-items, were developed for this study by two psychologists (AC, CN) with more than 30 years of experience with oncological patients. The items "I am at easy with my body"; "My body is a source of pleasure"; "Cancer humiliate my sensuality"; "Cancer turn out many of my passions" were rated on four 11-point visual analogue scales (VASs) ranging from "0" (=never) to "10" (=much). Lower scores on Comfort and Pleasure scales, and higher scores on Sensuality and Passion scales 
imply most discomfort for patients. The congruence between what we wanted to measure and the content of each item was judged by a group of expert psychologists. Pretesting with a pilot group proved that the items were easily understandable.

Sociodemographic and clinical information (i.e.: type of cancer, time since diagnosis, surgery) were also collected.

All measures were collected at baseline and 8 weeks later.

\section{Data reduction and statistical analysis}

To compare across variables with different upper and lower limits all the raw questionnaire and VAS scores were linearly transformed to $0-100$ scales, using the formula:

\section{Tscore $=(\mathrm{X}-\mathrm{Xmin}) / \mathrm{X}$ range $)^{\star} \mathrm{n}$}

where Tscore is the adjusted variable, $\mathrm{X}$ is the original variable, $\mathrm{X}_{\min }$ is the minimum observed value on the original variable and $\mathrm{X}_{\text {range }}$ is the difference between the maximum potential score and the minimum potential score on the original variable and $n$ is the upper limit of the rescaled variable.

The Tscores are characterized by a distribution with a mean of 50 and a standard deviation (SD) of 10 , with 0 and 100 assigned to the lowest and highest possible values, respectively.

For descriptive purpose we divided patients into two categories according to educational attainment $(<13$ years of education, and $>13$ years).

Based on the data available, comparisons have been made on different subsamples. (1) Dropout and no-dropout patients have been compared respect the baseline demographic and clinical characteristics, separately for TT and WL group $(\mathrm{N}=64)$. (2) To evaluate changes in psychological distress, anxiety, depression, qol, and feelings about one's body, data for patient with complete baseline and $2^{\text {nd }}$ assessment were compared, separately for TT and WL group $(n=47)$.

The statistical analyses included the Fisher exact test for categorical variables and the independent-samples $t$ test for continuous variables To assess the magnitude of the difference of mean scores between measures at different point of the study (baseline $=$ mean $1 ; 2^{\text {nd }}$ assessment, 8 weeks later=mean2) an estimate of the effect-size (ES) according to Cohen's coefficient $d$ was adopted [32]. Operationally the mean change in score is divided by the standard deviation (SD) of the first measure.

Formula: $\mathrm{d}=[($ mean2-mean1)/SD1 $]$

Differences greater than or equal to 0.2 are considered "small", greater than or equal to 0.5 "medium" and greater than or equal to 0.8 "large" [32].

All statistical analyses have been performed using STATA, version 11.0 (StataCorp, College Station, Tex). All tests were two-tailed.

\section{Results}

At baseline $8 \mathrm{WL}$ patients (11.11\%) returned incomplete questionnaires and were excluded by the analysis. There were completed data on 64 patients (TT, $n=36$ and $\mathrm{WL}, \mathrm{n}=28$ ), aged $36-73$ years (mean=52.48; $\mathrm{SD}=9.22$; median 52.01). Eleven patients had metastasis $(17.19 \%)$ that was localized in lymph nodes $(n=5 ; 45.45 \%)$, bones $(\mathrm{n}=3 ; 27.27 \%)$, liver $(\mathrm{n}=3 ; 27.27 \%)$. The mean time since diagnosis was 2.21 years $(\mathrm{SD}=2.28$; median 1.44 years). Thirty-six $(56.25 \%)$ patients reported significant distress (Distress Thermometer score >5), 21 (58.33\%) in the TT group and 15 (53.57\%) in the WL group.

Forty-seven patients completed baseline and $2^{\text {nd }}$ assessment questionnaires (73.44\%). Reasons noted for premature discontinuation were: hospitalization, fatigue, and other reasons, not further specified. As showed in Table 1, no differences emerged between dropout and no-dropout in sociodemographic and clinical characteristics among patients in TT or WL group.

In Table 2, means and SDs for the variables in study, at baseline (T0) and after 8 weeks (T1), are showed. Among TT group patients there were medium/small positive changes in Distress $(\mathrm{d}=0.53)$, Emotional qol $(\mathrm{d}=0.43)$, Social qol $(\mathrm{d}=0.37)$, Anxiety $(\mathrm{d}=0.34)$, Role qol $(\mathrm{d}=0.33)$ and Physical qol $(\mathrm{d}=0.21)$. Patients in the WL group have also reported a small positive change in Distress $(\mathrm{d}=0.33)$ and in Cognitive qol $(d=0.37)$, Comfort $(d=0.32)$ and Pleasure for one's body $(d=0.31)$ and a significant medium worsening in role $(\mathrm{ES}=0.54)$. About the perception of the Sensuality of one's body there was an improvement for patients in TT group, and a worsening for those in the WL.

During the tango-based therapy sessions the patients expressed issues related to their recent experience of cancer, to how it has deeply marked existential issues and the relationship with oneself and with the other, including relationships with the healthcare staff (Table 3).

\section{Discussion}

Tango therapy is considered part of DMTs, mind/body integrated forms of psychotherapy, usually offered as group therapy. DMTs combine the benefits of dance, movement, emotional expression, social support and creative activity in a single intervention. An approach in which patients learn to translate the sensory and affective cues into cognitions, verbalizations and new behaviours [8]. The methodological limitations of the studies that used DMT are mainly due of the lack of standardized protocol.

As already mentioned, some alternative therapies such as music (listening) and dance in addition to tango, and group psychotherapy have shown beneficial effects for patients and therefore the benefits showed in our results are partially limited or specific to Argentine tango. The specificity of the method used in this study is given by the availability of standardized, time-limited clinical protocol of Trossero for tango therapy $[23,24]$.

The imitative behaviours, interpersonal learning, cohesion, catharsis, existential reflections, exploration and processing of one's own personal experience through the interaction with others have had an impact on the effects observed. A factor that may have played a role in improving psychological conditions is related to connection and embrace. The intertwined steps, through somato-sensory perceptions, favour mutual connection, the recovery of a positive relationship with one's own body and that of the other, the focus on the here and now. The Argentine tango has been defined as an "embrace that you can dance" $[23,24]$ to underline the central role of the embrace and its effects in Argentine tango and tango therapy. It is a unique, containing embrace that promotes an intimate experience, listening to the other in a mutual exchange of emotions. The embrace is a primary behaviour that is activated after birth in response to situations of threat and stress or to comfort the infant. We know that the embrace stimulates the secretion of oxytocin, a neurotransmitter that promotes pro-social behaviours such as trust and connecting to others and is associated with a decrease in cortisol with the ensuing feelings of relaxation and happiness $[33,34]$. Another interpretation could be the therapeutic effect of group 
Table 1. Differences between dropout and no-dropout breast cancer patients in baseline demographic and clinical characteristics, separately for Tango-based Therapy group (TT) and Waiting list group (WL)

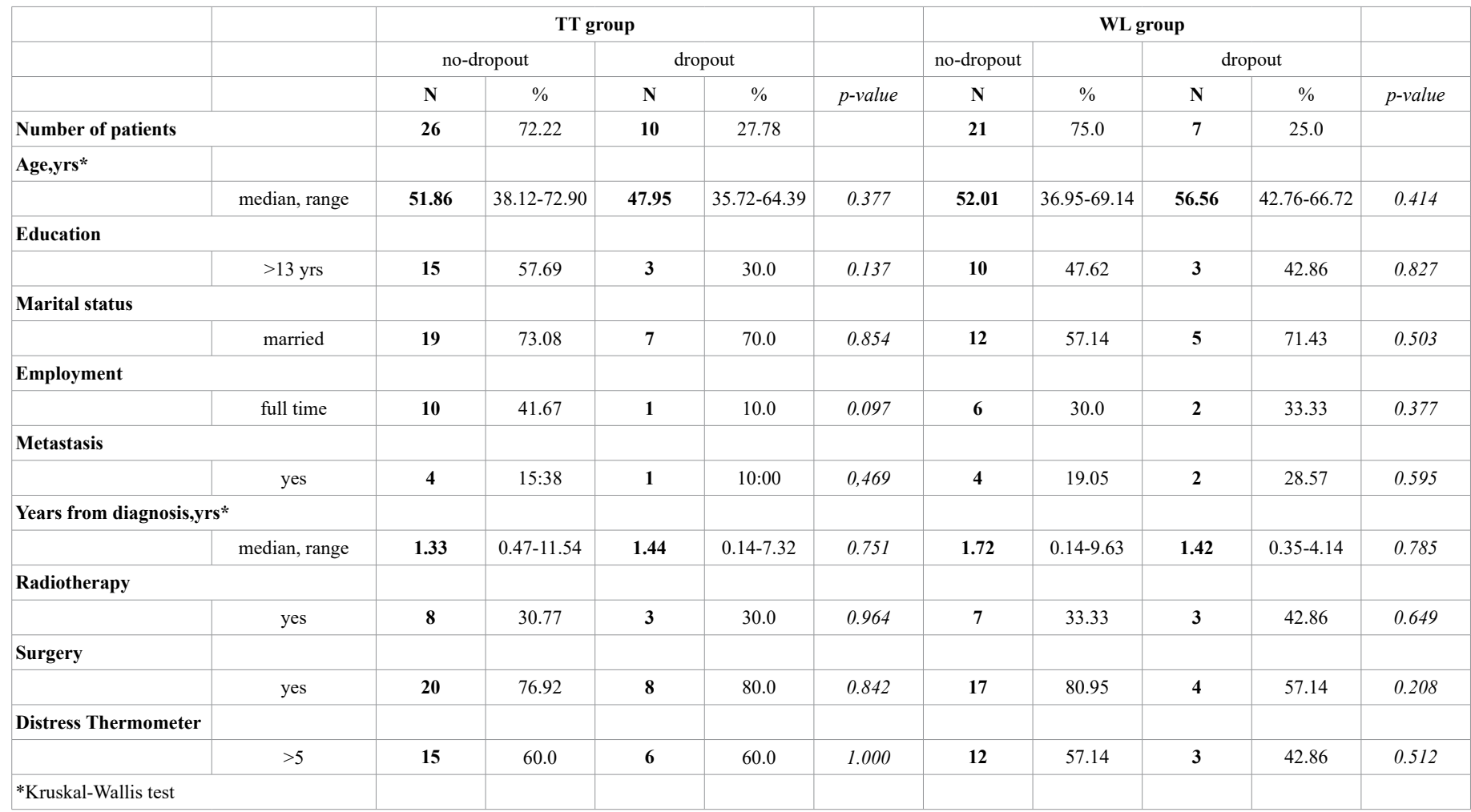

Table 2. Changes in Distress Thermometer, HADS-anxiety, HADS-depression, EORTC-domains and perception of one's body scores, separately for TT and WL group. Descriptive statistics are express as T-Score*

\begin{tabular}{|c|c|c|c|c|c|c|c|c|c|c|c|c|}
\hline & \multicolumn{4}{|c|}{ TT group $(n=26)$} & & \multicolumn{4}{|c|}{ WL group $(n=21)$} & \multirow[b]{3}{*}{$E S^{* *}$} & \multirow[b]{3}{*}{$\begin{array}{l}\text { p-value } \\
\text { (T0) }\end{array}$} & \multirow[b]{3}{*}{$\begin{array}{c}\text { p-value } \\
\text { (T1) }\end{array}$} \\
\hline & \multicolumn{2}{|c|}{ T0 assessment } & \multicolumn{2}{|c|}{$\mathrm{T} 1$ assessment } & \multirow[b]{2}{*}{$E S^{* *}$} & \multirow{2}{*}{\begin{tabular}{|} 
T0 assessment \\
mean
\end{tabular}} & \multirow[b]{2}{*}{ SD } & \multicolumn{2}{|c|}{$\mathrm{T} 1$ assessment } & & & \\
\hline & mean & SD & mean & SD & & & & mean & SD & & & \\
\hline DistressThermometer & 60.38 & 23.23 & 48.08 & 24.66 & 0.53 & 58.10 & 20.89 & 51.18 & 21.18 & 0.33 & 0.728 & 0.651 \\
\hline HADS-Anxiety & 43.04 & 16.30 & 37.55 & 14.59 & 0.34 & 41.95 & 15.33 & 40.90 & 13.89 & 0.07 & 0.816 & 0.428 \\
\hline HADS-Depression & 34.43 & 21.90 & 32.42 & 20.20 & 0.09 & 29.02 & 16.83 & 25.77 & 17.82 & 0.19 & 0.357 & 0.244 \\
\hline EORTC-Physical & 77.44 & 18.48 & 81.28 & 17.59 & 0.21 & 82.54 & 10.64 & 81.57 & 15.37 & 0.09 & 0.268 & 0.953 \\
\hline EORTC-Role & 71.15 & 23.36 & 78.85 & 24.75 & 0.33 & 84.92 & 15.73 & 76.47 & 24.34 & 0.54 & 0.026 & 0.743 \\
\hline EORTC-Cognitive & 78.21 & 23.46 & 80.13 & 17.65 & 0.08 & 79.37 & 24.10 & 88.24 & 17.45 & 0.37 & 0.869 & 0.123 \\
\hline EORTC-Emotional & 60.58 & 23.75 & 70.83 & 20.58 & 0.43 & 64.29 & 23.00 & 68.14 & 22.68 & 0.17 & 0.592 & 0.672 \\
\hline EORTC-Social & 69.23 & 26.12 & 78.85 & 22.39 & 0.37 & 79.37 & 22.92 & 77.45 & 23.53 & 0.08 & 0.169 & 0.836 \\
\hline EORTC-qol & 58.97 & 19.57 & 63.46 & 16.68 & 0.23 & 65.08 & 13.34 & 65.20 & 19.15 & 0.01 & 0.229 & 0.741 \\
\hline Comfort with one's body & 44.62 & 27.31 & 47.31 & 25.54 & 0.10 & 46.67 & 21.53 & 53.53 & 26.68 & 0.32 & 0.780 & 0.420 \\
\hline Pleasure with one's body & 42.31 & 24.38 & 45.77 & 23.52 & 0.14 & 37.62 & 21.43 & 45.88 & 19.38 & 0.31 & 0.493 & 0.986 \\
\hline Sensuality of one's body & 64.23 & 25.64 & 59.63 & 26.76 & 0.18 & 57.62 & 25.48 & 60.59 & 25.36 & 0.12 & 0.383 & 0.901 \\
\hline Passion for one's body & 52.69 & 29.61 & 53.46 & 26.82 & 0.03 & 46.19 & 32.48 & 48.82 & 31.20 & 0.08 & 0.427 & 0.586 \\
\hline
\end{tabular}

$*$ Tscore $=(\mathrm{X}-\mathrm{Xmin}) / \mathrm{X}$ range $)^{*} \mathrm{n}$ Tscore is the adjusted variable, $\mathrm{X}$ is the original variable, $\mathrm{Xmin}$ is the minimum observed value on the original variable and $\mathrm{Xrange}$ is the difference between the maximum potential score and the minimum potential score on the original variable and $\mathrm{n}$ is the upper limit of the rescaled variable, 100

$* * \mathrm{ES}=$ Cohen's $d$ Effect Size

*** independent-samples $t$ test by group

Example, to obtain the raw score starting from that T, for HADS subscale it will be sufficient to divide by 100 and then multiply by 21 - corresponding to the maximum theoretical score. For the distress thermometer that has a 0-10 response format, it will be sufficient to divide the T scores by 10. 
Table 3. How patients comment on the experience of Tango-based therapy

"The body awakens. The body that was only a source of suffering it vibrates with the heart, with the soul"

"Feel something coming back on. Here, there are ..."

"Having been so devastated and spending such a peaceful afternoon"

"But a moment of melancholy: I expected to be more fluid, I could not perceive the rhythm, I felt a little awkward"

"There are moments when the fear comes over me"

"Here I have not thought of anything"

"I felt like I was living again"

"Life is beautiful. With so much catastrophe around I had a moment of happiness"

"I didn't accept the disease and didn't want to walk"

"Then Dr. B. urged me, and here I am"

"My life is a disaster. Here I was happy: a moment of fun"

"I found the smile, even though we didn't know it, there was great harmony"

"I faced the illness with force, then I closed"

"This experience of the gaze, which is not superficial, but must create a contact, otherwise you cannot dance together, it puts you in connection with others.

"I felt affection embracing one that I do not know.

exercises focused on coordination, play and interpretation of music, which favour integration and social exchange. Group coordination of movements is associated with the pleasure and activation of the reward circuit even in the absence of a shared goal $[35,36]$. Listening to music can evoke emotions related to attachment such as love, joy and happiness [37]. Finally, tango - with its poetry, passion and deeply human themes $[24,38]$ has an impact on the autonomic nervous system (heart rate, blood pressure), on the muscular and emotional systems, as patients say: "Tango, with its music reaches depths that words can't get to", "The sound of Argentine Tango captivated and transformed me; it freed my mind and evoked a range of positive emotions".

Compared to the studies reported by Bradt et al. [8] that did not support the effect of DMT on body image, we have found an improvement in the perception of the Sensuality of one's body among TT patients while among WL patients this perception has worsened.

This result in TT group may be due to the characteristics of the Argentine tango, to its closed and restraining embrace, to the continuous and intimate psychophysical connection, which enhances somato-sensory perception and kinetic empathy [33,39]. This result could also be due to the choice of the dimensions most associated with the body image as "Feeling of Comfort and Pleasure for one's body" and "Perception of the Sensuality of one's body", or even to the choice of the visual analogue scale used more sensitive to register a change.

As well as the positive effect observed in the TT group with respect to anxiety and distress, only partially found in the Bradt review [40].

This study has some limitations. The first was not having compared Argentine tango with another type of consolidated intervention such as aerobic exercise, music or psychotherapy, a comparison that would have provided more reliable results. But organizational constraints led us to compare Argentine tango with a non-intervention (waiting list). Subsequent studies will be needed to confirm or not the effects of this study. The second limit concerns the number of patients who completed all 8 -weekly sessions. We have had a $26.56 \%$ attrition rate, with not significant differences between the two groups (TT $=25.00 \%$ vs $\mathrm{WL}=27.78 \%$ ). Although the proposed therapy is time-limited, the difficulty for cancer patients undergoing chemotherapy treatment is to maintain a commitment that lasts for 8 weeks, which involves moving every week to hospital and 90 minutes of exercise at each session.

\section{Conclusions}

Cancer has a 360-degree impact on a person's life causing physical, emotional and social suffering. Traditional cancer treatment is increasingly integrating rehabilitation interventions to improve the quality of life and strengthen personal resources. Creative art therapies constitute a valid therapeutic offer present today in the main cancer treatment centers. They promote recovery of the harmonious connection with body and emotional status; they help the patient to mitigate feelings of isolation, anxiety, depression, fear and more generally improve emotional distress.

The tango, with its music, its dance and its codes, acts as a facilitator and catalyst of emotions, memories and experiences inherent in the body and in the relationship with oneself and with others that can be elaborated in the group setting.

Tango therapy is a short, time-limited mind-body intervention. It is an active intervention, not a passive one as in the case of antidepressants or anxiety pills [41]. Unlike traditional psychotherapy, tango therapy is an intervention that is accessible to many, even to people with alexithymic traits or people who are less receptive to psychological interventions.

It has an impact on quality of life, it promotes self-acceptance, it enables contact with one's body, and allows being touched by others after cancer therapy. Cancer patients fear that their body has changed as a result of medical or surgical therapy and may hence be rejected. Therefore, the experience of embracing and intimacy, of respectful and empathic listening that is required in order to connect to the dance partner in the tango exercises, restores confidence and constitutes a precious opportunity for psycho-physical integration. From these preliminary data it seems that Trossero Tango therapy can constitute an effective treatment for psychological recovery in the oncological field.

\section{Authorship}

Anna Costantini lead Tango-based theraphy: conception and design of the study, drafting the article or revising it critically for important intellectual content, final approval of the version to be submitted.

Eva Mazzotti: conception and design of the study, analysis and interpretation of data, drafting the article or revising it critically for important intellectual content, final approval of the version to be submitted.

Serena Brunetti: conception of the study, acquisition of data, final approval of the version to be submitted.

Chiara Navarra co-lead Tango-based theraphy: conception and design of the study, revising it critically for important intellectual content, final approval of the version to be submitted. 
Paolo Marchetti: revising it critically, final approval of the version to be submitted.

Federico Trossero: conception of the study, revising it critically for important intellectual content, final approval of the version to be submitted.

\section{Declarations of interest}

None.

\section{Acknowledgements}

We thank all the patients who participated in this study.

\section{References}

1. Basso JC, Suzuki WA (2017) The Effects of acute exercise on mood, cognition, neurophysiology, and neurochemical pathways: A Review. Brain Plast 2: 127-152. [Crossref]

2. Cramp F, Byron-Daniel J (2012) Exercise for the management of cancer-related fatigue in adults. Cochrane Database Syst Rev 11: 1-10. [Crossref]

3. Höh JC, Schmidt T, Hübner J (2018) Physical activity among cancer survivors-what is their perception and experience? Support Care Cancer 26: 1471-1478. [Crossref]

4. Koelsch S (2012) Brain \& Music. Oxford: Wiley Blackwell.

5. Rudolph I, Scmidt T, Wozniak T (2018) Ballroom dancing as physical activity for patients with cancer: a systematic review and report of a pilot project. $J$ Cancer Res Clin Oncol 144: 759-770. [Crossref]

6. Brown S, Martinez MJ, Parsons LM (2006) The neural basis of human dance. Cereb Cortex 16: 1157-1167. [Crossref]

7. Boing L, Baptista F, Pereira GS (2017) Benefits of belly dance on quality of life, fatigue, and depressive symptoms in women with breast cancer - A pilot study of a nonrandomised clinical trial. $J$ Body Mov Ther 22: 460-466. [Crossref]

8. Bradt J, Shim M, Goodill SW (2015) Dance/movement therapy for improving psychological and physical outcome in cancer patients. Cochrane Database Syst Rev 1: 1-10. [Crossref]

9. Sturm I, Baak J, Storek B, Traore A, Thuss-Patience P (2014) Effect of dance on cancerrelated fatigue and quality of life. Support Care Cancer 22: 2241-2249. [Crossref]

10. Szalai M, Lévay B, Szirmai A, Papp I, Prémusz V, Bódis J (2015) A clinical study to assess the efficacy of belly dancing as a tool for rehabilitation in female patients with malignancies. Eur J Oncol Nurs 19: 60-65. [Crossref]

11. Aalbers S, Fusar-Poli L, Freeman RE (2017) Music therapy for depression. Cochrane Database Syst Rev 11. [Crossref]

12. Koelsh S, Offermanns K, Franzke P (2010) Music in the Treatment of Affective Disorders: An Exploratory Investigation of a New Method for Music-Therapeutic Research. Music Perception: An Interdisciplinary Journal 27: 307-316.

13. Alluri V, Toiviainen P, Jaaskelainen JP, Glerean E, Sams M, et al. (2012) Large-scale brain networks emerge from dynamic processing of music timbre, key and rhythm. Neuroimage 59: 3677-3689. [Crossref]

14. Pinninger R, Brown RF, Thorsteinsson ER, McKinley P (2012) Argentine tango dance compared to mindfulness meditation and waiting-list control: a randomised trial for treating depression. Complement Ther Med 20: 377-384 [Crossref]

15. Quiroga Murcia C, Bongard S, Kreutz G (2009) Emotional and Neurohumoral responses to dancing tango argentino: the effects of music and partner. Music and Medicine 1: 14-21.

16. Goodill SW (2018) Accumulating evidence for Dance/Movement Therapy in cancer care. Therapy in Cancer Care. Front Psychol 9: 1778. [Crossref]

17. Koch S, Kunz T, Likou S, Cruz R (2014) Effects of dance movement therapy and dance on health-related psychological outcomes: A meta-analysis. The Arts in Psychotherapy 41: 46-64.
18. Ho RT, Fong TC, Cheung IK, Yip PS, Luk MY (2016) Effects of a short-term dance movement therapy program on symptoms and stress in patients with breast cancer undergoing radiotherapy. A randomized, controlled, single-blind trial. J Pain Symptom Manage 51: 824-831. [Crossref]

19. Grassi L, Johansen C, Annunziata MA (2013) Screening for distress in cancer patients: a multicenter, nationwide study in Italy. Cancer 119: 1714-1721. [Crossref]

20. Wang T, Molassiotis A, Pui Man Chung B, Tan JY (2018) Unmet care needs of advanced cancer patients and their informal caregivers: a systematic review. BMC Palliative Care 17: 1-29. [Crossref]

21. Lotzke D, Ostermann T, Bussing A (2015) Argentine tango in Parkinson disease - a systematic review and meta-analysis. BMC Neurol 15: 226-244. [Crossref]

22. SNLG-ISS (2013) National system for guidelines of the higher institute of health Diagnosis and therapy of Parkinson's disease.

23. Trossero F (2006) Tango terapia. Buenos Aires: Coquena Ediciones.

24. Trossero F (2010) Tango terapia, fundamentos, metodologia, teoria y practica. Buenos Aires: Continente Ediciones Libro Buenos Aires

25. Bailey LM (1984) The use of songs in music therapy with cancer patients and their families. Music Therapy 4: 5-17.

26. Yun H, Sun L, Mao JJ (2009) Growth of integrative medicine at leading cancer center between 2009 and 2016: A systematic analysis of NCI-designated comprehensive cancer center websites. J Natl Cancer Inst Monogr 1: 1-10.

27. Wise TN, Biondi M, Costantini A (2013) Psycho-oncology. Washington, DC: American Psychiatric Publishing. pp 315-339.

28. Aaronson N, Ahmedzai S, Bergman B (1993) The European organisation for research and treatment of cancer QLQ-30: A quality of life instrument for use in international clinical trials in oncology. J Natl Cancer Inst 85: 365-376. [Crossref]

29. Zigmond AS, Snaith RP (1983) The hospital anxiety and depression scale. Acta Psychiatrica Scandinavica 67: 361-370.

30. Bjelland I, Dahl AA, Haug TT, Neckelmann D (2002) The validity of the hospita anxiety and depression scale. An update literature review. J Psychosom Res 52: 69-77. [Crossref]

31. Mitchell AJ, Meader N, Symonds P (2010) Diagnostic validity of the hospital anxiety and depression scale (HADS) in cancer and palliative settings: a meta-analisys. $J$ Affect Disord 126: 335-348. [Crossref]

32. Cohen J (1988) Statistical power analysis for the behavioral sciences. Hillsdale NJ: Lawrence Erlbaum Associates.

33. Grewen KM, Girdler SS, Amico J, Light KC (2005) Effects of partner support on resting oxytocin, cortisol, norepinephrine, and blood pressure before and after warm partner contact. Psychoso Med 67: 531-538. [Crossref]

34. Light KC, Grewen KM, Amico JA (2005) More frequent partner hugs and highe oxytocin levels are linked to lower blood pressure and heart rate in premenopausal women. Biol Psychol 69: 5-21. [Crossref]

35. Overy K, Molnar-Szakacs I (2009) Being together in time: musical experience and the mirror neuron system. Music Perception: An Interdisciplinary Journal 26: 489-504.

36. Wiltermuth S, Heath C (2009) Syncrony and cooperation. Psychol Sci 20: 1-5. [Crossref]

37. Cross I, Morley I (2008) The evolution of music: theories, definitions and the nature of the evidence. In: Malloch S, Trevarthen C [Eds], Communicative Musicality: Exploring the basis of Human Companionship. Oxford: Oxford University Press.

38. Tateo L (2014) The dialogical dance: self, identity construction, positioning and embodiment in tango dancers. Integr Psych Behav Sci 48: 299-321. [Crossref]

39. Khoene S, Dziobek I (2015) The role of interpersonal movement synchronisation in empathic functions: insights from Tango argentine and Capoeira. Int J Psychol 51:318 322. [Crossref]

40. Bradt J, Dileo C, Magill L, Teague A (2016) Music interventions for improving psychological and physical outcomes in cancer patients. Cochrane Database Syst Rev 8: 1-10. [Crossref]

41. Biondi M (2015) Pensieri terapeutici. Italia:Alpes

Copyright: $\odot 2020$ Costantini A. This is an open-access article distributed under the terms of the Creative Commons Attribution License, which permits unrestricted use, distribution, and reproduction in any medium, provided the original author and source are credited. 